\title{
Estimation of hepatocellular carcinoma mortality using aspartate aminotransferase to platelet ratio index
}

\author{
Kelvin Allenson, David Roife, Lillian S. Kao, Tien C. Ko, Curtis J. Wray \\ Department of Surgery, McGovern Medical School at the University of Texas Health Science Center at Houston, Houston, Texas, USA \\ Contributions: (I) Conception and design: K Allenson, CJ Wray; (II) Administrative support: TC Ko; (III) Provision of study materials or patients: \\ TC Ko, CJ Wray; (IV) Collection and assembly of data: D Roife, CJ Wray; (V) Data analysis and interpretation: K Allenson, LS Kao, CJ Wray; (VI) \\ Manuscript writing: All authors; (VII) Final approval of manuscript: All authors. \\ Correspondence to: Curtis J. Wray, MD, MS. Department of Surgery, Lyndon B. Johnson General Hospital, 5656 Kelley Street, Suite 30S62008, \\ Houston, Texas 77026, USA. Email: Curtis.J.Wray@uth.tmc.edu.
}

\begin{abstract}
Background: Hepatocellular carcinoma (HCC) patients with cirrhosis are high-risk for invasive procedures. Identification of those at risk may prevent complications and allow more informed decisionmaking. The aspartate aminotransferase (AST) to platelet ratio index (APRI) is a measure of cirrhosis that we hypothesize predicts survival and may estimate HCC mortality.

Methods: Institutional retrospective study of all HCC patients. Demographics and labs [bilirubin, international normalized ratio (INR), creatinine, AST and platelets] were recorded at the date-of-diagnosis to calculate APRI and the Model for End-Stage Liver Disease score (MELD). Poor survival was defined as death within 30-days from diagnosis. Models were created to determine predictors of death within 30-days and overall survival.
\end{abstract}

Results: A total of 829 patients comprised this study and <30-day death was observed in 111 patients (17\%). Mean APRI and MELD scores were higher in the $<30$-day death group. APRI [odds ratio (OR) 1.45, 95\% confidence interval (CI): 1.07-1.96] and MELD (OR 1.21, 95\% CI: 1.14-1.28) were predictive of <30-day death. Stratified by stage, APRI [hazard ratio (HR) 1.12, 95\% CI: 1.01-1.24] and MELD (HR 1.07, 95\% CI: 1.05-1.09) were associated with overall survival. Inclusion of APRI and MELD components in the Cox regression resulted in the best fit (c-index $=0.67$ ).

Conclusions: The APRI is an innovative marker of cirrhosis and survival for HCC patients. APRI provides additional prognostic information regarding the severity of cirrhosis and external validation is needed to determine clinical utility.

Keywords: Cirrhosis; liver cancer; survival estimation

Submitted Aug 30, 2018. Accepted for publication Nov 01, 2018.

doi: 10.21037/jgo.2018.11.01

View this article at: http://dx.doi.org/10.21037/jgo.2018.11.01

\section{Introduction}

Hepatocellular carcinoma (HCC) is the fifth leading cause of cancer death in the United States (1). Furthermore, the preponderance of hepatitis C-related HCC is unique to the United States and represents the fastest growing cause of cancer-related death in the country (2). Given that cirrhosis of any cause is present in $80-90 \%$ of HCC patients, ample time and opportunity exists to identify patients during the progression from fibrosis to cirrhosis and surveil this highrisk group for development of malignancy. In addition, for patients who have progressed to HCC, any means of riskstratification for invasive procedures has the potential to reduce procedure-related morbidity and mortality. The incidence of major complication following an invasive procedure, including liver biopsy, ablation, trans-arterial chemoembolization (TACE), or resection in the treatment of HCC patients has been observed between $1.3-50 \%$ of 


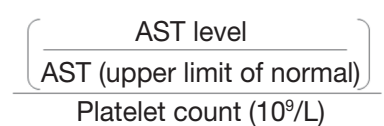

Figure 1 Mathematical equation to calculate the APRI. AST, aspartate aminotransferase; APRI, AST to platelet ratio index.

cases (3-6). In this context, non-invasive measures of liver function and cirrhosis have the ability to augment clinical decision making while sparing the risk and cost of biopsy.

At present, core needle biopsy is the gold standard assessment for liver fibrosis and cirrhosis $(7,8)$. Recent investigators have criticized the utility of biopsy as the tissue sampled is miniscule relative to the mass of the organ (9), the procedure is invasive in patients at risk for complications (4), and the histologic grade of the tissue sampled may not accurately reflect the more clinically relevant liver function (10). Alternatively, many surrogates exist for the noninvasive assessment of liver fibrosis. Fibrotest, Hepascore, Fibrometer, and the enhanced liver fibrosis (ELF) scores exploit the molecular intermediates of liver extracellular matrix turnover (10). Application is limited in that particular molecular targets as lab markers are not routinely clinically assessed. Aspartate aminotransferase (AST), alanine aminotransferase (ALT), platelet count, bilirubin, international normalized ratio (INR), and creatinine have traditionally been used to assess organ function in patients with liver disease and share the advantage of being routinely assessed lab metrics. These labs compose the AST to platelet ratio index (APRI), Fibrosis-4 (FIB-4) and Model for End-Stage Liver Disease (MELD) and have been widely studied in hepatitis B and C cohorts (10-20). Transient elastography, acoustic radiation force impulse and magnetic resonance elastography are different imaging modalities but all utilize the physical propagation of kinetic energy across the diseased, "stiff" liver $(21,22)$. Ability to detect cirrhosis is excellent but cost may be prohibitive and the widespread availability of these platforms is limited. Combination of the various scoring systems has been shown to improve the performance of predictive models, yet the clinical utility of detecting and measuring cirrhosis remains inconsistent.

The APRI amplifies the aberrations in the AST and platelet values that accompany cirrhosis (Figure 1). The AST to platelet ratio index amplifies the physiologic derangements reflected by AST and platelet count inherent to liver fibrosis and cirrhosis. As liver fibrosis proceeds, increasing liver inflammation is reflected by elevations in the AST level. This value is normalized according to the processing lab's normal to account for inter-lab variability. With compromised liver function, the liver's production of thrombopoietin declines and there is a subsequent decrease in platelet count. Therefore, as the AST numerator increases, and the platelet denominator decreases, higher APRI values will be obtained signifying a greater degree of liver cirrhosis. APRI has been shown to reliably identify cirrhotic from non-cirrhotic patients in the context of viral liver disease, and can predict the risk of developing viral-related HCC (23). Perhaps most compelling is that APRI has been shown to predict liver-related mortality independent of the well-established MELD or ChildPugh scores arguing that additional clinical data exists but is underutilized in the evaluation of such patients (24). Furthermore, patients with HCC and advanced cirrhosis are predisposed to poor survival and the APRI may allow for risk-stratification in order to prevent complications and allow for more informed and shared decision making (25). In this study we analyzed 829 patients in order to ascertain the predictive value of APRI for short term mortality as a means of risk-stratification prior to consideration for invasive procedure or surgery. We examined 30-day mortality as an estimate of procedure-related mortality (26).

\section{Methods}

\section{Patients}

We conducted an institutional review board approved retrospective study of all HCC patients diagnosed in a safety-net hospital system from 1998-2014. Standard demographics included age, stage at diagnosis, race, treatment (type of surgery, chemotherapy and radiation therapy), and mortality. In addition, laboratory values (bilirubin, INR, creatinine, albumin, AST and platelets) were recorded at the date of diagnosis to calculate APRI and MELD scores. The seventh version American Joint Committee on Cancer staging system was used to classify extent of disease.

\section{Statistical analyses}

Poor survival was defined as death within 30 days from the date of HCC diagnosis. Death within 30 days was recorded as a binary variable. A logistic regression model was created to determine predictors of 30-day death. Univariate analyses were completed with Student's $t$-test for continuous variables and chi-square analysis for categorical variables. Univariate 
logistic and multivariate logistic regression analyses were performed to determine predictors of $<30$-day survival. Receiver operating characteristic (ROC) curves were used to calculate the area under the curve (AUC) for the logistic regression models and compare the predictive power.

Overall survival was estimated using a multivariate Cox proportional hazards model. The final multivariate, multinomial model was created using the backward, stepwise method of covariate selection. The backward stepwise method was used to include only significant covariates ( $\mathrm{P}$ value of $<0.05$ ) in the final model. Significance of the exploratory model was tested using the Likelihood Ratio test to determine if the model is significantly better with additional predictor variables. Standard tests for regression diagnostics and assumptions, including tests of collinearity and interactions were performed. A covariate that did not meet the proportional hazards assumption was tested for possible inclusion as a time-varying covariate. This specifies a variable that varies continuously with respect to time. Confirming that a coefficient is time invariant is a way of testing the proportional hazards assumption. Proportional hazards imply that the relative hazard is fixed over time and this assumption would be violated if a time interaction proved significant. Iterations of the Cox proportional hazards model were compared for goodness of fit using Harrell's c-index. All statistical analyses were performed using Stata 14 (StataCorp, College Station, TX, USA).

\section{Results}

During the study period, 877 patients were diagnosed with HCC. A total of 48 patients (5.4\%) were excluded due to incomplete data. The remaining 829 HCC patients $(78 \%$ male) were included in this study. A total of 145 patients $(17.5 \%)$ survived less than 30 days. Mean age at diagnosis was 55.9 years [standard deviation (SD) 8.8] and there was no age difference between survival $<30$ and $\geq 30$ days (Table 1). There was no difference in hepatitis B virus (HBV) or hepatitis $\mathrm{C}$ virus (HCV) status between groups. Patients who died $<30$ days had liver dysfunction evidenced by increased APRI, MELD score, and alpha-fetoprotein (AFP) and decreased albumin.

A multivariate logistic regression model was created to identify factors associated with $<30$-day survival. APRI, creatinine, bilirubin, lower albumin values, higher AFP, advanced stage and no treatment were associated with $<30$-day survival (Table 2). The AUC of this model was 0.88 with APRI and 0.80 without the inclusion of APRI.
Since both stage and treatment are known to influence survival, the Cox proportional hazards model was stratified by these covariates. This demonstrated that higher APRI values [hazard ratio (HR) 1.15, 95\% confidence interval (CI): 1.03-1.30] were associated with worse overall survival. In addition, higher values of bilirubin, INR, creatinine and AFP were also predictors of poor survival (Table 3). There is a relatively weak direct relationship between APRI and MELD score (coefficient 0.32 , $\mathrm{P}$ value $<0.05,95 \%$ CI: $0.21-0.43)$, however, there was no interaction or evidence of collinearity in the Cox regression model. Inclusion of both APRI and MELD score components resulted in the highest c-index score (0.67).

\section{Discussion}

We investigated the relationship between APRI and clinical outcomes, <30-day survival as well as overall survival. In this study of 829 HCC patients, a lower APRI is associated with improved survival. Since APRI has previously been shown to correlate with the degree of cirrhosis, in this study it provided additional prognostic information, independent of variables that comprise the widely utilized MELD score. This noninvasive measure of cirrhosis may help clinicians estimate both cirrhosis and survival when considering different treatment options.

APRI was first proposed by Wai and colleagues as a biochemical surrogate for the diagnosis of advanced fibrosis and cirrhosis in patients with chronic hepatitis C (9). Since that time, the utility of the metric has further been explored with regard to concomitant HIV coinfection, and also hepatitis B cohorts with continued favorable performance at identifying cirrhotics from non-cirrhotics $(20,24)$. More recently, APRI has been shown to be predictive of the risk of developing HCC in the setting of established cirrhosis and additionally can predict liver-related mortality after hepatic resection for HCC (11-13). The current study adds to the body of APRI literature since this study cohort is significant larger and more diverse than those described in earlier reports.

In our study we explored the ability of APRI to predict the risk of early mortality in HCC patients. We believe such an application to be appropriate as the majority of the patients in our cohort exhibited viral hepatitis, with $66.2 \% \mathrm{HCV}$ positive, and $16.2 \% \mathrm{HBV}$ positive. Whereas the majority of APRI studies focused on the diagnosis of cirrhosis, we explored the association between APRI and early mortality. It is important to assess short term mortality 
Table 1 Standard patient cohort demographics ( $\mathrm{N}=829)$

\begin{tabular}{|c|c|c|c|}
\hline $\begin{array}{l}\text { Patient } \\
\text { demographics } \\
(\mathrm{N}=829)\end{array}$ & $\begin{array}{l}\text { Mortality } \\
<30 \text { days }\end{array}$ & $\begin{array}{l}\text { Mortality } \\
>30 \text { days }\end{array}$ & $P$ value \\
\hline Age (SD) & 55.9 (9.3) yrs & 55.9 (8.7) yrs & 0.98 \\
\hline Gender & & & 0.004 \\
\hline Male & 126 (86.9\%) & $519(75.8 \%)$ & \\
\hline Female & $19(13.1 \%)$ & $165(24.1 \%)$ & \\
\hline Race & & & 0.463 \\
\hline Caucasian & $48(33.1 \%)$ & $199(29.1 \%)$ & \\
\hline African-American & $49(33.7 \%)$ & $210(30.7 \%)$ & \\
\hline Hispanic & $34(23.4 \%)$ & $193(28.2 \%)$ & \\
\hline Asian & $14(9.7 \%)$ & $82(12 \%)$ & \\
\hline HBV & & & 0.940 \\
\hline Positive & $23(15.9 \%)$ & $111(16.2 \%)$ & \\
\hline Negative & 110 (75.9\%) & $541(79 \%)$ & \\
\hline $\mathrm{HCV}$ & & & 0.918 \\
\hline Positive & $96(66.2 \%)$ & 457 (70.1\%) & \\
\hline Negative & $43(29.7 \%)$ & 209 (30.6\%) & \\
\hline AFP (SD) & $\begin{array}{c}80,597.01 \\
(182,220.9)\end{array}$ & $\begin{array}{c}15,241.62 \\
(77,195.21)\end{array}$ & $<0.0001$ \\
\hline Total bilirubin (SD) & $5.48(6.83)$ & $1.99(2.95)$ & $<0.0001$ \\
\hline INR (SD) & $1.67(0.75)$ & $1.34(0.7)$ & $<0.0001$ \\
\hline Albumin (SD) & $2.34(0.68)$ & $2.85(0.71)$ & $<0.0001$ \\
\hline AST (SD) & 331.4 (342.6) & $153.8(295.7)$ & $<0.0001$ \\
\hline Platelets (SD) & $193.5(127.3)$ & $181.7(129.3)$ & 0.33 \\
\hline Creatinine (SD) & $1.43(1.03)$ & $0.97(0.48)$ & $<0.0001$ \\
\hline APRI (SD) & $6.38(7.22)$ & $3.52(9.75)$ & 0.002 \\
\hline MELD (SD) & $19.0(8.2)$ & $11.7(4.5)$ & $<0.0001$ \\
\hline Stage & & & $<0.0001$ \\
\hline I & $9(6.2 \%)$ & 143 (20.9\%) & \\
\hline II & $11(7.6 \%)$ & $125(18.3 \%)$ & \\
\hline III & $38(26.2 \%)$ & $182(26.6 \%)$ & \\
\hline IV & 75 (51.7\%) & $200(29.2 \%)$ & \\
\hline Unknown & $12(8.3 \%)$ & $33(4.8 \%)$ & \\
\hline Treatment & & & $<0.0001$ \\
\hline Yes & $12(8.3 \%)$ & 364 (53.2\%) & \\
\hline No & $122(84.1 \%)$ & $283(41.4 \%)$ & \\
\hline
\end{tabular}

Table 1 (continued)
Table 1 (continued)

\begin{tabular}{lccc}
\hline Variables & $\begin{array}{c}\text { Mortality } \\
<30 \text { days }\end{array}$ & $\begin{array}{c}\text { Mortality } \\
>30 \text { days }\end{array}$ & P value \\
\hline $\begin{array}{c}\text { Surgery } \\
\text { Yes }\end{array}$ & $4(2.8 \%)$ & $63(9.2 \%)$ & 0.011 \\
No & $130(89.7 \%)$ & $581(84.9 \%)$ & \\
Chemotherapy & & & $<0.0001$ \\
Yes & $5(3.4 \%)$ & $204(29.8 \%)$ & \\
No & $129(89 \%)$ & $440(64.3 \%)$ & \\
Radiotherapy & & & 0.065 \\
Yes & $1(0.7 \%)$ & $25(3.7 \%)$ & \\
No & $133(91.7 \%)$ & $616(90.1 \%)$ & \\
\hline
\end{tabular}

HBV, hepatitis B virus; HCV, hepatitis C virus; AFP, alphafetoprotein; INR, international normalized ratio; AST, aspartate aminotransferase; APRI, AST to platelet ratio index; MELD, model for end stage liver disease.

Table 2 Logistic regression model to identify variables associated with less than 30-day survival

\begin{tabular}{lccc}
\hline Variables & OR & P value & $95 \% \mathrm{Cl}$ \\
\hline APRI & 1.33 & 0.03 & $1.03-1.71$ \\
Creatinine & 2.21 & 0.00 & $1.53-3.18$ \\
Total bilirubin & 1.13 & 0.00 & $1.06-1.21$ \\
Albumin & 0.55 & 0.01 & $0.36-0.84$ \\
INR & 1.28 & 0.10 & $0.96-1.71$ \\
Stage & & & \\
2 & 2.22 & 0.17 & $0.71-6.96$ \\
3 & 2.33 & 0.10 & $0.86-6.29$ \\
4 & 6.50 & 0.00 & $2.47-17.13$ \\
Unknown & 3.53 & 0.27 & $0.37-33.67$ \\
AFP & 1.00 & 0.04 & $1.00-1.00$ \\
Treatment & 0.16 & 0.00 & $0.08-0.33$ \\
\hline
\end{tabular}

OR, odds ratio; $\mathrm{Cl}$, confidence interval; APRI, aspartate aminotransferase to platelet ratio index; INR, international normalized ratio; AFP, alpha-fetoprotein. 
Table 3 Cox proportional hazards model to identify variables associated with overall survival

\begin{tabular}{lccc}
\hline Variables & HR & P value & $95 \% \mathrm{Cl}$ \\
\hline APRI & 1.15 & 0.02 & $1.03-1.30$ \\
Albumin & 0.69 & 0.00 & $0.60-0.80$ \\
Total bilirubin & 1.03 & 0.01 & $1.01-1.05$ \\
INR & 1.22 & 0.00 & $1.07-1.38$ \\
Creatinine & 1.27 & 0.00 & $1.11-1.45$ \\
AFP & 1.12 & 0.00 & $1.08-1.15$ \\
Hepatitis & & & \\
HBV & 0.97 & 0.86 & $0.66-1.40$ \\
HCV & 0.98 & 0.89 & $0.76-1.27$ \\
HBV/HCV & 0.62 & 0.22 & $0.29-1.32$ \\
Unknown & 4.13 & 0.00 & $1.59-10.72$ \\
\hline
\end{tabular}

Stratified by stage and treatment. HR, hazard ratio; CI, confidence interval; APRI, aspartate aminotransferase to platelet ratio index; INR, international normalized ratio; AFP, alphafetoprotein; HBV, hepatitis B virus; HCV, hepatitis $\mathrm{C}$ virus.

risk as means of risk stratification prior to consideration of an invasive procedure, biopsy and/or surgery $(27,28)$. As applied, we observed a $<30$-day mortality of $17.5 \%$ suggesting that risk stratification should be considered in high-risk HCC patient cohorts. As previously mentioned, our regression model exhibited the best performance when combining APRI data with other available laboratory data, namely components of MELD score and serum albumin $(10,24)$. Of note, the individual components of the MELD score (bilirubin, INR and creatinine) were included in our regression model. Using MELD score as a continuous variable would have meant inclusion of already transformed data (natural logarithm of bilirubin, INR and creatinine).

The Child-Pugh score has historically been used to assess the prognosis of cirrhotic patients undergoing surgery. More recently, the MELD score was used for further prognostication initially for cirrhotic patients undergoing transjugular intrahepatic portosystemic shunt (TIPS) procedures, and subsequently for the prioritization of liver allografts for transplantation (29). MELD has become near ubiquitous in the evaluation of liver function (30). Interestingly, both are composites scores, which mirror the attempts from recent studies to combine APRI with other available metrics to improve performance. In our study, we observed that APRI can predict mortality independent of MELD score components, suggesting that available information exists but is underutilized in assessing patients with hepatic cirrhosis and compromised liver function. Since the labs composing APRI and MELD are routinely assessed together, and the MELD score itself requires calculation, we recommend that all available data be considered in order to properly assess risk in cirrhotic HCC patients prior to intervention.

Our study included a diverse racial and ethnic composition and with a high prevalence of hepatitis C. Demographic variables more consistent with the challenges of $\mathrm{HCV}$ related cirrhosis and HCC, compared to large majority-HBV cohorts that frequently have less severe cirrhosis secondary to the different mechanisms of hepatocarcinogenesis. However, our efforts are not without limitation. While our cohort is the largest to date to examine the relationship between APRI and survival outcomes, it is retrospective in nature. Although, medical management of cirrhosis and invasive treatment of HCC evolved over the survey period, accounting for this in the multivariate model did not affect the results. Additionally, since this cohort hails from a safety-net facility, liver transplantation is not included in the treatment algorithm of these patients. At present, APRI is a noninvasive measure that may aid clinicians when counseling cirrhotic HCC patients about the risks and benefits of various invasive treatments. Further study is needed to determine the clinical utility of using APRI for prognostic and treatment decisions.

\section{Conclusions}

APRI is associated with both the diagnosis of cirrhosis and HCC across multiple high-risk populations. In our study, we demonstrate that APRI is predictive of mortality in HCC patients, independent of the MELD score. APRI provides additional prognostic information in the management of HCC patients, and may be used to stratify high-risk patients when considering invasive procedures.

\section{Acknowledgments}

Funding: American Cancer Society MRSG-12-178-01PCSM

\section{Footnote}

Conflicts of Interest: The authors have no conflicts of interest to declare. 
Ethical Statement: The authors are accountable for all aspects of the work in ensuring that questions related to the accuracy or integrity of any part of the work are appropriately investigated and resolved. Retrospective collection of patient data was approved by the UT Health and Lyndon B Johnson General Hospital Institutional Review Board.

Open Access Statement: This is an Open Access article distributed in accordance with the Creative Commons Attribution-NonCommercial-NoDerivs 4.0 International License (CC BY-NC-ND 4.0), which permits the noncommercial replication and distribution of the article with the strict proviso that no changes or edits are made and the original work is properly cited (including links to both the formal publication through the relevant DOI and the license). See: https://creativecommons.org/licenses/by-ncnd/4.0\%.

\section{References}

1. Siegel RL, Miller KD, Jemal A. Cancer statistics, 2018. CA Cancer J Clin 2018;68:7-30.

2. El-Serag HB. Hepatocellular carcinoma. N Engl J Med 2011;365:1118-27.

3. Rockey DC, Caldwell SH, Goodman ZD, et al. Liver biopsy. Hepatology 2009;49:1017-44.

4. Hackl C, Schlitt HJ, Renner P, et al. Liver surgery in cirrhosis and portal hypertension. World J Gastroenterol 2016;22:2725-35.

5. Facciorusso A, Serviddio G, Muscatiello N. Local ablative treatments for hepatocellular carcinoma: An updated review. World J Gastrointest Pharmacol Ther 2016;7:477-89.

6. Doussot A, Lim C, Lahat E, et al. Complications after Hepatectomy for Hepatocellular Carcinoma Independently Shorten Survival: A Western, Single-Center Audit. Ann Surg Oncol 2017;24:1569-78.

7. Lahat E, Eshkenazy R, Zendel A, et al. Complications after percutaneous ablation of liver tumors: a systematic review. Hepatobiliary Surg Nutr 2014;3:317-23.

8. Martin J, Khatri G, Gopal P, et al. Accuracy of ultrasound and noninvasive markers of fibrosis to identify patients with cirrhosis. Dig Dis Sci 2015;60:1841-7.

9. Wai CT, Greenson JK, Fontana RJ, et al. A simple noninvasive index can predict both significant fibrosis and cirrhosis in patients with chronic hepatitis C. Hepatology 2003;38:518-26.

10. Martinez SM, Crespo G, Navasa M, et al. Noninvasive assessment of liver fibrosis. Hepatology 2011;53:325-35.

11. Hann HW, Wan S, Lai Y, et al. Aspartate aminotransferase to platelet ratio index as a prospective predictor of hepatocellular carcinoma risk in patients with chronic hepatitis B virus infection. J Gastroenterol Hepatol 2015;30:131-8.

12. Hung HH, Su CW, Lai CR, et al. Fibrosis and AST to platelet ratio index predict post-operative prognosis for solitary small hepatitis B-related hepatocellular carcinoma. Hepatol Int 2010;4:691-9.

13. Ichikawa $T$, Uenishi $T$, Takemura $S$, et al. A simple, noninvasively determined index predicting hepatic failure following liver resection for hepatocellular carcinoma. J Hepatobiliary Pancreat Surg 2009;16:42-8.

14. Ishizuka M, Kubota K, Kita J, et al. Aspartate aminotransferase-to-platelet ratio index is associated with liver cirrhosis in patients undergoing surgery for hepatocellular carcinoma. J Surg Res 2015;194:63-8.

15. Lin CS, Chang CS, Yang SS, et al. Retrospective evaluation of serum markers APRI and AST/ALT for assessing liver fibrosis and cirrhosis in chronic hepatitis $\mathrm{B}$ and $\mathrm{C}$ patients with hepatocellular carcinoma. Intern Med 2008;47:569-75.

16. Shaheen AA, Myers RP. Diagnostic accuracy of the aspartate aminotransferase-to-platelet ratio index for the prediction of hepatitis C-related fibrosis: a systematic review. Hepatology 2007;46:912-21.

17. Sharma S, Khalili K, Nguyen GC. Non-invasive diagnosis of advanced fibrosis and cirrhosis. World J Gastroenterol 2014;20:16820-30.

18. Shen SL, Fu SJ, Chen B, et al. Preoperative aspartate aminotransferase to platelet ratio is an independent prognostic factor for hepatitis B-induced hepatocellular carcinoma after hepatic resection. Ann Surg Oncol 2014;21:3802-9.

19. Yu J, Liang P, Yu XL, et al. Needle track seeding after percutaneous microwave ablation of malignant liver tumors under ultrasound guidance: analysis of 14-year experience with 1462 patients at a single center. Eur J Radiol 2012;81:2495-9.

20. Xiao G, Yang J, Yan L. Comparison of diagnostic accuracy of aspartate aminotransferase to platelet ratio index and fibrosis-4 index for detecting liver fibrosis in adult patients with chronic hepatitis $B$ virus infection: a systemic review and meta-analysis. Hepatology 2015;61:292-302.

21. Huwart L, Sempoux C, Vicaut E, et al. Magnetic resonance elastography for the noninvasive staging of liver fibrosis. Gastroenterology 2008;135:32-40. 
22. Kim BK, Kim SU, Kim HS, et al. Prospective validation of FibroTest in comparison with liver stiffness for predicting liver fibrosis in Asian subjects with chronic hepatitis B. PLoS One 2012;7:e35825.

23. Abdelgawad IA. Clinical utility of simple non-invasive liver fibrosis indices for predicting hepatocellular carcinoma (HCC) among Egyptian patients. J Clin Pathol 2015;68:154-60.

24. Nunes D, Fleming C, Offner G, et al. Noninvasive markers of liver fibrosis are highly predictive of liverrelated death in a cohort of $\mathrm{HCV}$-infected individuals with and without HIV infection. Am J Gastroenterol 2010;105:1346-53.

25. Harvin JA, Van Buren G, Tsao K, et al. Hepatocellular carcinoma survival in uninsured and underinsured patients. J Surg Res 2011;166:189-93.

26. Mayo SC, Shore AD, Nathan H, et al. Refining the definition of perioperative mortality following

Cite this article as: Allenson K, Roife D, Kao LS, Ko TC, Wray CJ. Estimation of hepatocellular carcinoma mortality using aspartate aminotransferase to platelet ratio index. J Gastrointest Oncol 2020;11(2):291-297. doi: 10.21037/ jgo.2018.11.01 hepatectomy using death within 90 days as the standard criterion. HPB (Oxford) 2011;13:473-82.

27. Wray CJ, Harvin JA, Silberfein EJ, et al. Pilot prognostic model of extremely poor survival among high-risk hepatocellular carcinoma patients. Cancer 2012;118:6118-25.

28. Kao WY, Chiou YY, Hung HH, et al. Risk factors for long-term prognosis in hepatocellular carcinoma after radiofrequency ablation therapy: the clinical implication of aspartate aminotransferase-platelet ratio index. Eur J Gastroenterol Hepatol 2011;23:528-36.

29. Cholongitas E, Papatheodoridis GV, Vangeli M, et al. Systematic review: The model for end-stage liver disease--should it replace Child-Pugh's classification for assessing prognosis in cirrhosis? Aliment Pharmacol Ther 2005;22:1079-89.

30. Schroeder RA, Marroquin CE, Bute BP, et al. Predictive indices of morbidity and mortality after liver resection.

Ann Surg 2006;243:373-9. 